\title{
A STUDY OF STREAMLINES IN SECOND GRADE FLUID FLOWS
}

\author{
C.S. Bagewadi* \& S. Bhagya**
}

\begin{abstract}
We obtain solutions for second grade fluid in $(\phi, \psi)$ net where $\phi(x, y)=$ constant, an arbitrary family of curves and $\psi(x, y)=$ constant, stream lines. Further exact solutions are determined when the stream line patterns are of the form

$$
\frac{y-g(x)}{f(x)}=\text { constant or } \frac{x-k(y)}{m(y)}=\text { constant. }
$$
\end{abstract}

Keywords: Streamlines, Second grade fluid, Incompressible.

\section{Introduction}

Non-Newtonian fluids have gained more and more important industrially, over the past decades. Polymer solutions and polymer melts are the most common examples of non-Newtonian fluids. The equations of motion of such fluids are highly nonlinear and one order higher than the Navier-Stokes equations. Martin [1] has used a natural curvilinear co-ordinate system $(\phi, \psi)$ in the physical plane $(x, y)$ where $\psi=$ constant are the streamlines and $\phi=$ constant is an arbitrary family of curves

- Department of Studies in Mathematics, Kuvempu University, Jnana Sahyadri, Shimoga.577 451, Kornataka., India.

** Department of Mathematics, P.E.S. College, Bangalore - 560 050, India. 
to study plane viscous flows. C.S. Bagewadi and Siddabasappa [6] studied the plane rotating viscous $M H D$ flows by using differential geometry techniques. O.P. Chandna and Labropulu [2] obtained the exact solutions for steady plane viscous flows by taking the arbitrary family of curves $\phi=$ constant to be $x=$ constant. Rajagopal [3] found some interesting exact solutions of unsteady unidirectional second grade fluid flows. More recently, Labropulu [4] studied generalized Beltrami flows and other closed form solutions of an unsteady viscoelastic fluid.

In the present work we first use Martin's [1] method to decompose the basic equations of non-Newtonian fluids and next following the work of Labropulu and O.P. Chandna [5], A.M. Siddiqui, P.N. Kaloni and O.P. Chandna [7], C.S. Bagewadi and S. Bhagya [9] and Erikson J.L [11], we study whether the second grade fluid flow along a given family of curves $\frac{y-g(x)}{f(x)}=$ constant, where $f(x) \neq 0$ can exist?

The plan of this paper is as follows; in section 2, we employ Martin's [I] approach and recast the flow equations. This section also contains the recasting of the equations in a new form by employing some results from differential geometry. In section 3 , we outline the method of determining whether a given family of curves can be the streamlines. In section 4, we deal with the particular examples.

\section{Equation of Motions}

The flow of a homogeneous incompressible second grade fluid flow, neglecting thermal effects and body forces, is governed by [10]

$$
\begin{aligned}
& \operatorname{div} V=0 \\
& \operatorname{div} T=\rho V
\end{aligned}
$$

where $T$ is Cauchy stress which describes second grade fluids given by B.D. Coleman and W. Noll [8],

$$
T=-p 1+\mu A_{1}+\alpha_{1} A_{2}+\alpha_{2} A_{1}^{2}
$$

The following nomenclatures are used; $V$ the velocity vector field, $p$ the fluid pressure function, $\rho$ the constant fluid density, $\mu$ the constant coefficient of viscosity and $\alpha$, and $\alpha_{2}$ are the normal stress moduli.

The Rivlin-Ericksen tensors $A_{1}$ and $A_{2}$ are defined as

$$
\begin{aligned}
& A_{1}=\left(\operatorname{grad} V+\left(\operatorname{grad} V^{+}\right.\right. \\
& A_{2}=A_{1}+\left(\operatorname{grad} V^{\prime} A_{1}+A_{1}(\operatorname{grad} V)\right.
\end{aligned}
$$


Here (grad V)' denotes the transpose of grad V. If we substitute (3) in (2) and make use of (4) we get

$$
\begin{aligned}
& -\operatorname{grad} \rho+\mu \nabla^{2} V+\alpha_{1} \mid \nabla^{2} V_{1}+\nabla^{2}(\nabla \times V) \times V+\operatorname{grad}\left(V \cdot \nabla^{2} V+\frac{1}{4}\left|A_{1}\right|^{2}\right) \\
& +\left(\alpha_{1}+\alpha_{2}\right) \operatorname{div} A_{1}^{2}=\rho V
\end{aligned}
$$

where $\nabla^{2}$ denotes the Laplacian, $V_{t}$ denotes the partial derivative of $V$ with respect to time $t$ and

$$
\left|A_{1}\right|^{2}=\operatorname{tr} A_{1} A_{1}^{\dagger}
$$

The decomposition of equations (1) and (2) gives

$$
\begin{gathered}
\frac{\partial u}{\partial x}+\frac{\partial v}{\partial y}=0 \\
u \frac{\partial u}{\partial x}+v \frac{\partial u}{\partial y}+\frac{1}{\rho} \frac{\partial p}{\partial x}=\frac{\mu}{\rho u_{0}}\left(\frac{\partial^{2} u}{\partial x^{2}}+\frac{\partial^{2} u}{\partial y^{2}}\right)+\frac{\alpha_{1}}{\rho}\left\{\frac { \partial } { \partial x } \left(2 u \frac{\partial^{2} u}{\partial x^{2}}+2 v \frac{\partial^{2} u}{\partial x \partial y}+4\left(\frac{\partial u}{\partial x}\right)^{2}\right.\right. \\
\left.\left.+2 \frac{\partial v}{\partial x}\left(\frac{\partial v}{\partial x}+\frac{\partial u}{\partial y}\right)\right)+\frac{\partial}{\partial y}\left[\left(u \frac{\partial}{\partial x}+v \frac{\partial}{\partial y}\right)\left(\frac{\partial v}{\partial x}+\frac{\partial u}{\partial y}\right)+2 \frac{\partial u}{\partial x} \frac{\partial u}{\partial y}+2 \frac{\partial v}{\partial x} \frac{\partial v}{\partial y}\right]\right\} \\
+\frac{\alpha_{2}}{\rho}\left\{\frac{\partial}{\partial x}\left(4\left(\frac{\partial u}{\partial x}\right)^{2}+\left(\frac{\partial v}{\partial x}+\frac{\partial u}{\partial y}\right)^{2}\right)\right\} \\
u \frac{\partial v}{\partial x}+\frac{1}{\partial y} \frac{\partial p}{\partial y}=\frac{\mu}{\rho u_{0}}\left(\frac{\partial^{2} v}{\partial x^{2}}+\frac{\partial^{2} x z}{\partial y^{2}}\right)+\frac{\alpha_{1}}{\rho}\left\{\frac { \partial } { \partial x } \left[\left(u \frac{\partial}{\partial x}+v \frac{\partial}{\partial y}\right)\left(\frac{\partial v}{\partial x}+\frac{\partial u}{\partial y}\right)+2 \frac{\partial u}{\partial x} \frac{\partial u}{\partial y}\right.\right. \\
\left.\left.+2 \frac{\partial v}{\partial x} \frac{\partial v}{\partial y}\right]+\frac{\partial}{\partial y}\left(2 u \frac{\partial^{2}}{\partial x \partial y}+2 v \frac{\partial^{2} v}{\partial y^{2}}+4\left(\frac{\partial v}{\partial y}\right)^{2}+2 \frac{\partial u}{\partial y}\left(\frac{\partial v}{\partial x}+\frac{\partial u}{\partial y}\right)\right)\right\} \\
+
\end{gathered}
$$


We introduce the Vorticity function $\omega$ and energy function $h$ as

$$
\omega=\frac{\partial r}{\partial x}-\frac{\partial u}{\partial y}
$$

and $\quad h=p+\frac{1}{2} \rho\left(u^{2}+v^{2}\right)-\alpha_{1}\left(u \nabla^{2} u+v \nabla^{2} v\right)-\left(\frac{3 \alpha_{1}+2 \alpha_{2}}{4}\right)\left|A_{1}\right|^{2}$

where $\quad \nabla^{2}=\frac{\partial^{2}}{\partial x^{2}}+\frac{\partial^{2}}{\partial y^{2}}$

and

$$
\left|A_{1}\right|^{2}=4\left(\frac{\partial u}{\partial x}\right)^{2}+4\left(\frac{\partial v}{\partial y}\right)^{2}+2\left(\frac{\partial u}{\partial y}+\frac{\partial v}{\partial x}\right)^{2}
$$

We write the equations $(7)$ to $(10)$ as system of second order equations in non dimensional variables by using (9) and (10) in (7) and (8) and taking non dimensional quantities for velocity and pressure

$$
\begin{aligned}
& \frac{v}{u_{0}}=(u(x, y), v(x, y), 0), p(x, y)=\frac{p}{\rho u_{0}^{2}} \text { as follows: } \\
& \frac{\partial h}{\partial x}=v \omega-\frac{1}{R} \frac{\partial \omega}{\partial y}-\frac{W e}{R} v\left(\frac{\partial^{2} \omega}{\partial x^{2}}+\frac{\partial^{2} \omega}{\partial y^{2}}\right) \\
& \frac{\partial h}{\partial y}=-u \omega+\frac{1}{R} \frac{\partial \omega}{\partial x}+\frac{W e}{R} v\left(\frac{\partial^{2} \omega}{\partial x^{2}}+\frac{\partial^{2} \omega}{\partial y^{2}}\right) \\
& h=p+\frac{1}{2}\left(u^{2}+v^{2}\right)-\frac{W e}{R}\left[v\left(\frac{\partial^{2} u}{\partial x^{2}}+\frac{\partial^{2} u}{\partial y^{2}}\right)+v\left(\frac{\partial^{2} v}{\partial x^{2}}+\frac{\partial^{2} v}{\partial y^{2}}\right)\right] \\
& -\left(\frac{3+2 v}{2}\right) \frac{W e}{R}\left[2\left(\frac{\partial u}{\partial x}\right)^{2}+2\left(\frac{\partial v}{\partial y}\right)^{2}+\left(\frac{\partial v}{\partial x}+\frac{\partial u}{\partial y}\right)^{2}\right]
\end{aligned}
$$

Here $u, v$ are the velocity components and $p$ the pressure function of $x, y$ and $R=\frac{\rho_{0}}{\mu}$ the Reynolds number, $W e=\frac{\alpha_{1} v_{0}}{\mu}$ is the Weissenberg number, $v=\frac{\alpha_{2}}{\alpha_{1}}$ is the ratio of normal stress moduli and $v_{0}$ the characterstic velocity. Thus (6), (9), 
(11), (12) and (13) are five equations in five unknown functions $u, v, \omega, h$ and $p$ of $x, y$.

The continuity equation (6) implies the existence of a stream function $\psi(x, y)$ such that

$$
u=\frac{\partial \psi}{\partial y}, \quad v=-\frac{\partial \psi}{\partial x}
$$

We introduce a curvilinear coordinate system in the physical plane in which the curves $\psi(x, y)=$ constant are the streamlines and the curves $\phi(x, y)=$ constant are arbitrary.

Let

$$
x=x(\phi, \psi), \quad y=y(\phi, \psi)
$$

define a curvilinear net in the $(x, y)$ plane with the squared element of arc length along any curves given by

$$
d s^{2}=E(\phi, \psi) d \phi^{2}+2 F(\phi, \psi) d \phi d \psi+G(\phi, \psi) d \psi^{2}
$$

where

$$
E=\left(\frac{\partial x}{\partial \phi}\right)^{2}+\left(\frac{\partial y}{\partial \phi}\right)^{2}, \quad F=\frac{\partial x}{\partial \phi} \frac{\partial x}{\partial \psi}+\frac{\partial y}{\partial \phi} \frac{\partial y}{\partial \psi}, \quad G=\left(\frac{\partial x}{\partial \psi}\right)^{2}+\left(\frac{\partial y}{\partial \psi}\right)^{2}
$$

Equation (15) can be solved to obtain $\phi=\phi(x, y), \psi=\psi(x, y)$ such that

$$
\begin{array}{ll}
\frac{\partial x}{\partial \phi}=J \frac{\partial \psi}{\partial y}, & \frac{\partial x}{\partial \psi}=-J \frac{\partial \phi}{\partial y} \\
\frac{\partial y}{\partial \phi}=-J \frac{\partial \psi}{\partial x}, & \frac{\partial y}{\partial \psi}=J \frac{\partial \phi}{\partial x}
\end{array}
$$

provided $0<|J|<\infty$, and $J$ is Jacobian given by

$$
J=\frac{\partial x}{\partial \phi} \frac{\partial y}{\partial \psi}-\frac{\partial x}{\partial \psi} \frac{\partial y}{\partial \phi}= \pm \sqrt{E G-F^{2}}= \pm W
$$

Denoting by $\alpha$ the local angle of inclination of the tangent to the coordinate line $\psi=$ constant, directed in the sense of increasing $\phi$, we have from differential geometry the following [1]: 


$$
\begin{aligned}
& \frac{\partial x}{\partial \phi}=\sqrt{E} \cos \alpha, \quad \frac{\partial y}{\partial \phi}=\sqrt{E} \sin \alpha \\
& \frac{\partial x}{\partial \psi}=\frac{F}{\sqrt{E}} \cos \alpha-\frac{j}{\sqrt{E}} \sin \alpha \\
& \frac{\partial y}{\partial \psi}=\frac{F}{\sqrt{E}} \sin \alpha+\frac{J}{\sqrt{E}} \cos \alpha \\
& \frac{\partial \alpha}{\partial \phi}=\frac{J}{E} \Gamma_{11}^{2} \quad \frac{\partial \alpha}{\partial \psi}=\frac{J}{E} \Gamma_{12}^{2} \\
& K=\frac{1}{W}\left[\frac{\partial}{\partial \psi}\left(\frac{W}{E} \Gamma_{11}^{2}\right)-\frac{\partial}{\partial \phi}\left(\frac{W}{E} \Gamma_{12}^{2}\right)\right]=0
\end{aligned}
$$

where

$$
\begin{aligned}
& \Gamma_{11}^{2}=\frac{1}{2 W^{2}}\left[-F \frac{\partial E}{\partial \phi}+2 E \frac{\partial F}{\partial \phi}-E \frac{\partial E}{\partial \psi}\right] \\
& \Gamma_{12}^{2}=\frac{1}{2 W^{2}}\left[E \frac{\partial G}{\partial \phi}-F \frac{\partial E}{\partial \psi}\right] \\
& \Gamma_{22}^{2}=\frac{1}{2 W^{2}}\left[E \frac{\partial G}{\partial \psi}-2 F \frac{\partial F}{\partial \psi}+F \frac{\partial G}{\partial \phi}\right]
\end{aligned}
$$

and $K$ is the Gaussian curvature.

We transform equations (9) to (13) governing our flow into new forms in the new variables $\phi, \psi$.

\section{Equation of continuity and Vorticity}

Martin [1] has obtained the necessary and sufficient condition for the flow of a fluid, along the co-ordinate lines $\psi=$ constant of a curvilinear co-ordinate system (15) with $d^{2} s^{2}$ given by (16) to satisfy the principle of conservation of mass to be

$$
W q=\sqrt{E}, \quad u+i v=\frac{\sqrt{E}}{W} e^{i \alpha}
$$


where

$$
\begin{aligned}
& \alpha=\int \frac{\partial \alpha}{\partial \phi} d \phi+\frac{\partial \alpha}{\partial \psi} d \psi \\
& =\int \frac{J}{E}\left(\Gamma_{11}^{2} d \phi+\Gamma_{12}^{2} d \psi\right)
\end{aligned}
$$

Then equation (12) becomes

$$
\omega=\frac{1}{W}\left[\frac{\partial}{\partial \phi}\left(\frac{F}{W}\right)-\frac{\partial}{\partial \psi}\left(\frac{E}{W}\right)\right]
$$

In this work, we consider that the fluid towards higher or lower parameter values $\phi$ accordingly as $J$ is positive or negative and speed $q$ of the fluid flow is given in (25).

\section{Linear Momentum Equation}

On employing (14) in the linear momentum equations (11), (12) and making use of (18), we have

$$
\begin{aligned}
& R J \frac{\partial h}{\partial \phi}=F \frac{\partial \omega}{\partial \phi}-E \frac{\partial \omega}{\partial \dot{\psi}} \\
& R J\left(\frac{\partial h}{\partial \psi}+\omega\right)=G \frac{\partial \omega}{\partial \phi}-F \frac{\partial \omega}{\partial \psi}+W e J \nabla^{2} \omega
\end{aligned}
$$

\section{Energy Equation}

Employing (14) in the energy equation (13), transforming to $(\phi, \psi)$ net and using (18), we have

$$
\begin{gathered}
h=p+\frac{E}{2 W^{2}}-\frac{W e}{R W^{2}}\left(F \frac{\partial \omega}{\partial \phi}-E \frac{\partial \omega}{\partial \psi}\right)-\left(\frac{3+2 v}{2}\right) \frac{W e}{R}\left\{\omega^{2}+\frac{4}{W \sqrt{E}}\left[\frac{\partial}{\partial \psi}\left(\frac{\sqrt{E}}{W}\right) \Gamma_{11}^{2}\right.\right. \\
\left.\left.-\frac{\partial}{\partial \phi}\left(\frac{\sqrt{E}}{W}\right) \Gamma_{12}^{2}\right]\right\}
\end{gathered}
$$


Summing up, we have

Theorem 1: If the streamlines $\psi(x, y)=$ constant of a steady plane motion of an incompressible second grade fluid are taken as one set of co-ordinate lines in a curvilinear co-ordinate system $\phi, \psi$ in the physical plane, then the flow is governed by the system of equations (25) to (29).

These are five equations in six unknown functions $E, F, G, \omega, h$ and $p$ of $\phi, \psi$. Here

$$
\nabla^{2} \omega=\frac{1}{W}\left[\frac{\partial}{\partial \phi}\left(\frac{G \frac{\partial \omega}{\partial \phi}-F \frac{\partial \omega}{\partial \psi}}{W}\right)+\frac{\partial}{\partial \psi}\left(\frac{-F \frac{\partial \omega}{\partial \phi}+E \frac{\partial \omega}{\partial \psi}}{W}\right)\right]
$$

Eliminating $h$ from the linear momentum equations by using the integrability conditions $\frac{\partial^{2} h}{\partial \phi \partial \psi}=\frac{\partial^{2} h}{\partial \psi \partial \partial \phi^{\prime}}$, we have

$$
\begin{array}{ll}
W \nabla^{2} \omega \mp R \frac{\partial \omega}{\partial \phi} \pm W e \frac{\partial}{\partial \phi}\left(\nabla^{2} \omega\right)=0 & \text { (Integrability) } \\
\omega=\frac{1}{W}\left[\frac{\partial}{\partial \phi}\left(\frac{F}{W}\right)-\frac{\partial}{\partial \psi}\left(\frac{E}{W}\right)\right] & \text { Norticity) } \\
\frac{\partial}{\partial \psi}\left(\frac{W}{E} \Gamma_{11}^{2}\right)-\frac{\partial}{\partial \phi}\left(\frac{W}{E} \Gamma_{12}^{2}\right)=0 & \text { (Gauss) }
\end{array}
$$

of three equations for $E, F, G$ and $\omega$ as function of $\phi, \psi$. The continuity equation (25) determines the speed $q=q(\phi, \psi)$, the linear momentum equations determines

$$
h=h(\phi, \psi)=\int \frac{\partial h}{\partial \phi} d \phi+\frac{\partial h}{\partial \psi} d \psi
$$

and the energy equation provides the pressure function $p=p(\phi, \psi)$. 


\section{A Study of a Family of Curves in Van Mises Co-ordinate System}

In this section we study the properties of streamlines in Von Mises co-ordinate system that is in $(x, \psi)$ and $(y, \psi)$ coordinate net.

\subsection{For the $(x, \psi)$ Co-ordinate Net}

To analyze whether a given family of curves $\frac{y-g(x)}{f(x)}=$ constant can or cannot be the streamlines, we assume the affirmative so that there exists some function $M(\psi)$ such that

$$
\frac{y-g(x)}{f(x)}=M(\psi), \quad M^{\prime}(\psi) \neq 0
$$

where $M^{\prime}(\psi)$ is the derivative of unknown function $M(\psi)$. The system of equations (31) can be now determined for these flows by setting $\phi(x, y)=x=$ constant, so that the curvilinear co-ordinate net is the Von Mises net $x, \psi$. Employing equation (32) in (16) and simplifying the resulting equation, we obtain

$$
\begin{aligned}
& d s^{2}=\left\{1+[g(x)+f(x) M(\psi)]^{2} d \phi^{2}+2[g(x)+f(x) M(\psi)] f(x) M(\psi) d \phi d \psi\right. \\
& +f^{2}(x) M^{2}(\psi) d \psi^{2}
\end{aligned}
$$

Equations (17) and (19) become

$$
\begin{aligned}
& E=1+\left[g^{\prime}(x)+f^{\prime}(x) M(\psi)\right]^{2} \\
& F=\left|g^{\prime}(x)+f^{\prime}(x) M(\psi)\right| f(x) M^{\prime}(\psi), \\
& G=f^{\prime}(x) M^{\prime 2}(\psi) \text { and } J=W=f(x) M^{\prime}(\psi)
\end{aligned}
$$

so that

$$
E=1+\frac{F^{2}}{G}
$$

when the fluid is assumed to be flowing in the direction of increasing $x$ along the streamlines. Employing (33) in (31), Gauss equation is identically satisfied. If the curves (32) constitute a streamline pattern for a steady plane flow of an incompressible second grade fluid, then the flow must satisfy the Vorticity function 


$$
\begin{aligned}
& \omega=\frac{1}{f^{2}(x) M^{\prime}(\psi)}\left\{f(x) g^{\prime \prime}(x)-2 g^{\prime}(x) f^{\prime}(x)+\left[f(x) f^{\prime \prime}(x)-2 f^{\prime 2}(x)\right] M(\psi)\right. \\
& \left.+\left[1+g^{\prime 2}(x)\right] \frac{M^{\prime \prime}(\psi)}{M^{\prime 2}(\psi)}+2 g^{\prime}(x) f^{\prime}(x) \frac{M(\psi) M^{\prime \prime}(\psi)}{M^{\prime 2}(\psi)}+f^{\prime 2}(x) \frac{M^{2}(\psi) M^{\prime \prime}(\psi)}{M^{\prime 2}(\psi)}\right\}
\end{aligned}
$$

and

$f(x) M^{\prime}(\psi) \frac{\partial^{2} \omega}{\partial x^{2}}-2\left[g^{\prime}(x)+f^{\prime}(x) M(\psi)\right] \frac{\partial^{2} \omega}{\partial x \partial \psi}+\frac{1}{f(x) M^{\prime}(\psi)}\left[1+f^{\prime 2}(x)\right.$ $\left.+2 f^{\prime}(x) g^{\prime}(x) M(\psi)+f^{\prime 2}(x) M^{2}(\psi)\right] \frac{\partial^{2} \omega}{\partial \psi^{2}}+\frac{1}{f(x)}\left\{2 g^{\prime}(x) f^{\prime}(x)-f(x) g^{\prime \prime}(x)+\right.$ $+\left[2 f^{\prime 2}(x)-f(x) f^{\prime \prime}(x)\right] M(\psi)-\left[1+g^{\prime 2}(x)\right] \frac{M^{\prime \prime}(\psi)}{M^{\prime 2}(\psi)}-2 g^{\prime}(x) f^{\prime}(x) \frac{M(\psi) M^{\prime \prime}(\psi)}{M^{\prime 2}(\psi)}$ $\left.-f^{\prime 2}(x) \frac{M^{2}(\psi) M^{\prime \prime}(\psi)}{M^{\prime 2}(\psi)}\right\} \frac{\partial \omega}{\partial \psi}-R \frac{\partial \omega}{\partial x}+\frac{W e}{f(x) M^{\prime}(\psi)}\left(f(x) M^{\prime}(\psi) \frac{\partial^{3} \omega}{\partial x^{3}}-2 / g^{\prime}(x)\right)$ $\left.+f^{\prime}(x) M(\psi)\right] \frac{\partial^{2} \omega}{\partial x^{2}} \frac{\partial \omega}{\partial \psi}+\frac{1}{f(x) M^{\prime}(\psi)}\left[1+g^{\prime 2}(x)+2 g^{\prime}(x) f^{\prime}(x) M(\psi)\right.$ $\left.+f^{\prime 2}(x) M^{2}(\psi)\right] \frac{\partial \omega}{\partial x} \frac{\partial^{2} \omega}{\partial \psi^{2}}+\frac{1}{g(x)}\left\{4 g^{\prime}(x) f^{\prime}(x)-3 f(x) g^{\prime \prime}(x)+\left[4 f^{2}(x)\right.\right.$ $\left.-3 f(x) f^{\prime \prime}(x)\right] M(\psi)-\left[1+g^{\prime 2}(x)\right] \frac{M^{\prime \prime}(\psi)}{M^{\prime 2}(\psi)}-2 f^{\prime}(x) g^{\prime}(x) \frac{M(\psi) M^{\prime \prime}(\psi)}{M^{\prime 2}(\psi)}$ $\left.-f^{\prime 2}(x) \frac{M^{2}(\psi) M^{\prime \prime}(\psi)}{M^{\prime 2}(\psi)}\right\} \frac{\partial^{2} \omega}{\partial x \partial \psi}+\frac{2}{f^{2}(x) M^{\prime}(\psi)}\left\{f(x) g^{\prime}(x) g^{\prime \prime}(x)-f^{\prime}(x)\left[1+g^{\prime 2}(x)\right]\right.$ $+\left[f(x) f^{\prime}(x) g^{\prime \prime}(x)+f(x) g^{\prime}(x) f^{\prime \prime}(x)-2 g^{\prime}(x) f^{\prime 2}(x)\right] M(\psi)+f^{\prime}(x)\left[f(x) f^{\prime \prime}(x)\right.$ $\left.\left.-f^{\prime 2}(x)\right] M^{2}(\psi)\right\} \frac{\partial^{2} \omega}{\partial \psi^{2}}+\frac{1}{f^{2}(x)}\left\{f(x)\left[3 f^{\prime}(x) g^{\prime \prime}(x)+2 g^{\prime}(x) f^{\prime \prime}(x)\right]-4 g^{\prime}(x) f^{\prime 2}(x)\right.$ $-f^{2}(x) g^{\prime \prime \prime}(x)+\left[5 f(x) f^{\prime}(x) f^{\prime \prime}(x)-4 f^{\prime 3}(x)-f^{2}(x) f^{\prime \prime \prime}(x)\right] M(\psi)+2 \mid f^{\prime}(x) g^{\prime 2}(x)$ 


$$
\begin{aligned}
& \left.+f^{\prime}(x)-f(x) g^{\prime}(x) g^{\prime \prime}(x)\right] \frac{M^{\prime \prime}(\psi)}{M^{\prime 2}(\psi)}+2\left[2 g^{\prime}(x) f^{\prime 2}(x)-f(x) g^{\prime}(x) f^{\prime \prime}(x)\right. \\
& \left.\left.\left.-f(x) f^{\prime}(x) g^{\prime \prime}(x)\right] \frac{M(\psi) M^{\prime \prime}(\psi)}{M^{\prime 2}(\psi)}+2 f^{\prime}(x)\left[f^{\prime 2}(x)-f(x) f^{\prime \prime}(x)\right] \frac{M^{2}(\psi) M^{\prime \prime}(\psi)}{M^{\prime 2}(\psi)}\right\} \frac{\partial \omega}{\partial \psi}\right)=0
\end{aligned}
$$

and $M(\psi)$ is some function of such that $M^{1}(\psi) \neq 0$.

Conculsion: A given family of curves $\frac{y-g(x)}{f(x)}=$ constant is a permissible family of streamlines in second grade fluids if and only if the solution obtained for $M(\psi)$ is such that $M^{1}(\psi) \neq 0$.

\subsection{For the $(y, \psi)$ co-ordinate net}

To analyze whether a given family of curves $\frac{x-k(y)}{m(y)}=$ constant can or cannot be streamlines, we assume the affirmative so that there exists some function $N(\psi)$ such that

$$
\frac{x-k(y)}{m(y)}=N(\psi), \quad N^{\prime}(\psi) \neq 0 .
$$

where $N^{\prime}(\psi)$ is the derivative of the unknown function $N(\psi)$ and we take the coordinate lines $\phi=$ constant to be $y=$ constant. Employing equation (36) in (16) and simplifying the resulting equation, we obtain

$$
\begin{aligned}
d s^{2}= & {\left[1+\left(k^{\prime}(y)+m^{\prime}(y) N(\psi)\right)^{2}\right] d \phi^{2}+2\left[k^{\prime}(y)+m^{\prime}(y) N(\psi)\right] m(y) N^{\prime}(\psi) d \phi d \psi } \\
& +m^{2}(y) N^{\prime 2}(\psi) d^{2} \psi
\end{aligned}
$$

Equations (17) and (19) become

$$
\begin{array}{ll}
E=\left\{1+\left(k^{\prime}(y)+m^{\prime}(y) N(\psi)\right)^{2}\right\}, & F=\left[k^{\prime}(y)+m^{\prime}(y) N(\psi)\right] m(y) N^{\prime}(\psi) \\
G=m^{2}(y) N^{\prime 2}(\psi) & W=\sqrt{E G-F^{2}}=m(y) N^{\prime}(\psi)
\end{array}
$$

when the fluid is assumed to be flowing in the direction of increasing $y$ - along the streamlines. Employing (38) in (31), Gauss equation is identically satisfied.

If the curves (36) constitute a streamline pattern for a steady plane flow of an incompressible second grade fluid, then the flow must satisfy 


$$
\begin{aligned}
\omega & =\frac{1}{m^{2}(y) N^{\prime}(\psi)}\left[m(y) k^{\prime \prime}(y)-2 k^{\prime}(y) m^{\prime}(y)+\left(m(y) m^{\prime \prime}(y) 22 m^{\prime 2}(y)\right) N(\psi)\right. \\
& \left.+\left[1+k^{\prime 2}(y)\right] \frac{N^{\prime \prime}(\psi)}{N^{\prime 2}(\psi)}+2 k^{\prime}(y) m^{\prime}(y) \frac{N(\psi) N^{\prime \prime}(\psi)}{N^{\prime 2}(\psi)}+m^{\prime 2}(y) \frac{N^{2}(\psi) N^{\prime \prime}(\psi)}{N^{\prime 2}(\psi)}\right]
\end{aligned}
$$

and

$m(y) N^{\prime}(\psi) \frac{\partial^{2} \omega}{\partial y^{2}}-2\left[k^{\prime}(y)+m^{\prime}(y) N(\psi)\right] \frac{\partial^{2} \omega}{\partial y \partial \psi}+\frac{1}{m(y) N^{\prime}(\psi)}\left[1+k^{\prime 2}(y)+2 k^{\prime}(y) m^{\prime}(y) N(\psi)\right.$

$\left.+m^{\prime 2}(y) N^{2}(\psi)\right]\left[\frac{\partial^{2} \omega}{\partial \psi^{2}}+\frac{1}{m(y)} 2 k^{\prime}(y) m^{\prime}(y)-m(y) k^{\prime \prime}(y)+\left[2 m^{\prime 2}(y)-m(y) m(y) m^{\prime \prime}(y)\right] N(\psi)\right.$

$\left.-\left[1+k^{\prime 2}(y)\right] \frac{N^{\prime \prime}(\psi)}{N^{\prime 2}(\psi)}-2 k^{\prime}(y) m^{\prime}(y) \frac{N(\psi) N^{\prime \prime \prime}(\psi)}{N^{\prime 2}(\psi)}-m^{\prime 2}(y) \frac{N^{2}(\psi) N^{\prime \prime}(\psi)}{N^{\prime 2}(\psi)}\right\} \frac{\partial \omega}{\partial \psi}$

$-R \frac{\partial \omega}{\partial y}+\frac{W_{e}}{m(y) N^{\prime}(\psi)}\left(m(y) N^{\prime}(\psi) \frac{\partial^{2} \omega}{\partial y^{2}}-2\left[k^{\prime}(y)+m^{\prime}(y) N(\psi)\right] \frac{\partial^{2} \omega}{\partial y^{2}} \frac{\partial \omega}{\partial \psi}\right.$

$+\frac{1}{m(y) N^{\prime}(\psi)}\left[1+k^{\prime 2}(y)+2 k^{\prime}(y) m^{\prime}(y) N(\psi)+m^{2}(y) N^{2}(\psi)\right] \frac{\partial \omega}{\partial y} \frac{\partial^{2} \psi}{\partial \psi^{2}}+\left\{\frac{1}{m(y)} 4 k^{\prime}(y) m^{\prime}(y)\right.$

$-3 m(y) k^{\prime \prime}(y)+\left[4 m^{12}(y)-3 m(y) m^{\prime \prime}(y)\right] N(\psi)-\left[1+k^{\prime 2}(y)\right] \frac{N^{\prime \prime}(\psi)}{N^{\prime 2}(\psi)}$

$\left.-2 k^{\prime}(y) m^{\prime}(y) \frac{N(\psi) N^{\prime \prime}(\psi)}{N^{\prime 2}(\psi)}-m^{\prime 2}(y) \frac{N^{2}(\psi) N^{\prime \prime}(\psi)}{N^{\prime 2}(\psi)}\right\} \frac{\partial^{2} \omega}{\partial y \partial \psi}+\frac{2}{m^{2}(y) N^{\prime \prime}(\psi)}\left\{m(y) k^{\prime}(y) k^{\prime \prime}(y)\right.$

$-m^{\prime}(y)\left[1+k^{\prime 2}(y)\right]+\left[m(y) m^{\prime}(y) k^{\prime \prime}(y)+m(y) k^{\prime}(y) m^{\prime \prime}(y)-2 k^{\prime}(y) m^{\prime 2}(y)\right] N(\psi)$

$\left.+m^{\prime}(y)\left[m(y) m^{\prime \prime}(y)-m^{\prime 2}(y)\right] N^{2}(\psi)\right\} \frac{\partial^{2} \omega}{\partial \psi^{2}}+\frac{1}{m^{2}(y)}\left\{m(y)\left[3 m^{\prime}(y) k^{\prime \prime}(y)+2 k^{\prime}(y) m^{\prime \prime}(y)\right]\right.$

$-4 k^{\prime}(y) m^{\prime 2}(y)-m^{2}(y) k^{\prime \prime}(y)+\left[5 m(y) m^{\prime}(y) m^{\prime \prime}(y)-4 m^{\prime 3}(y)-m^{2}(y) m^{\prime \prime \prime}(y)\right] N(\psi)$ 
$+2\left[m^{\prime}(y) k^{2}(y)+m^{\prime}(y)-m(y) k^{\prime}(y) k^{\prime \prime}(y)\right] \frac{N^{\prime \prime}(\psi)}{N^{2}(\psi)}+2\left[2 k^{\prime}(y) m^{\prime 2}(y)-m(y) k^{\prime}(y) m^{\prime \prime}(y)\right.$

$\left.\left.\left.-m(y) m^{\prime}(y) k^{\prime \prime}(y)\right] \frac{N(\psi) N^{\prime \prime}(\psi)}{N^{\prime 2}(\psi)}+2 m^{\prime}(y)\left[m^{\prime 2}(y)-m(y) m^{\prime \prime}(y)\right] \frac{N^{2}\left(\psi / N^{\prime \prime}(\psi)\right.}{N^{\prime 2}(\psi)}\right] \frac{\partial \omega}{\partial \psi}\right)=0$

and $N(\psi)$ is some function of $\psi$ such that $N^{\prime}(\psi) \neq 0$.

Conclusion: A given family of curves $\frac{x-k(y)}{m(y)}=$ constânt is a permissible family of streamlines in second grade fluids if and only if the solution obtained for $N(\psi)$ is such that $N^{\prime}(\psi) \neq 0$.

\section{Applications:}

Example 1. (Flow with $y-C_{1} x^{2}-C_{2} x+1=$ constant as streamlines). We assume

$$
y=C_{1} \dot{x}^{2}+C_{2} x+M(\psi) ; \quad M^{\prime}(\psi) \neq 0
$$

where $C_{1}, C_{2}$ are arbitrary constants and comparing this $y$ with (32), we have $g(x)=C_{1} x^{2}+C_{2} x, \quad f(x)=1$

The streamline pattern for this flow is shown in Figure 1.

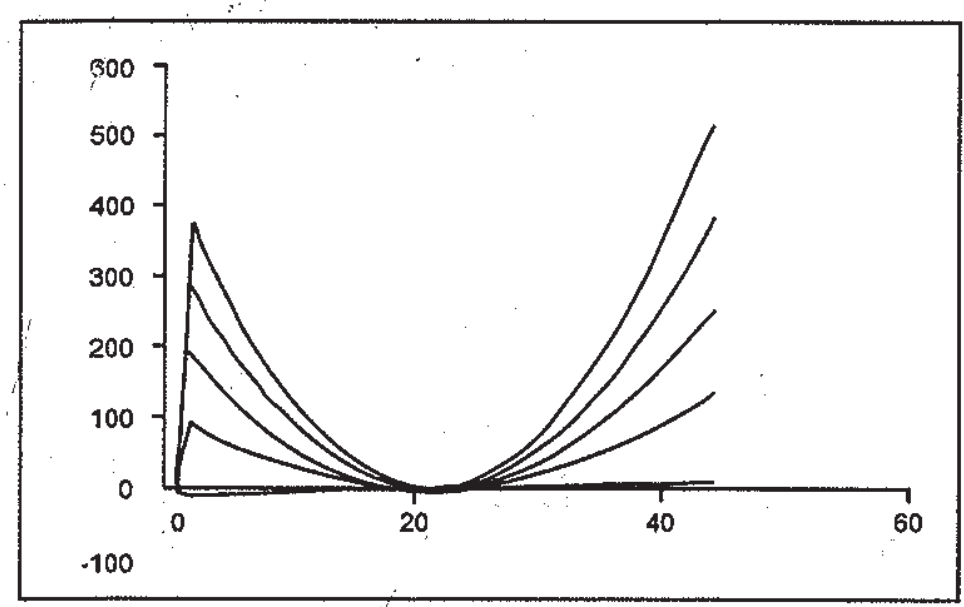

Figure 1. Streamline, pattern for $y-C_{1} x^{2}-C_{2} x+1=$ constant. 
Example 2. (Flow with $y-C_{1} e^{x}-C_{2} x=$ constant as streamlines). We assume

$$
y=C_{1} e^{x}+C_{2} x+M(\psi) ; \quad M^{\prime}(\psi) \neq 0
$$

where $C_{1}, C_{2}$ are arbitrary constants and therefore, we have

$$
g(x)=C_{1} e^{x}+C_{2} x, \quad f(x)=1
$$

The streamline pattern for this flow is shown in Figure 2.

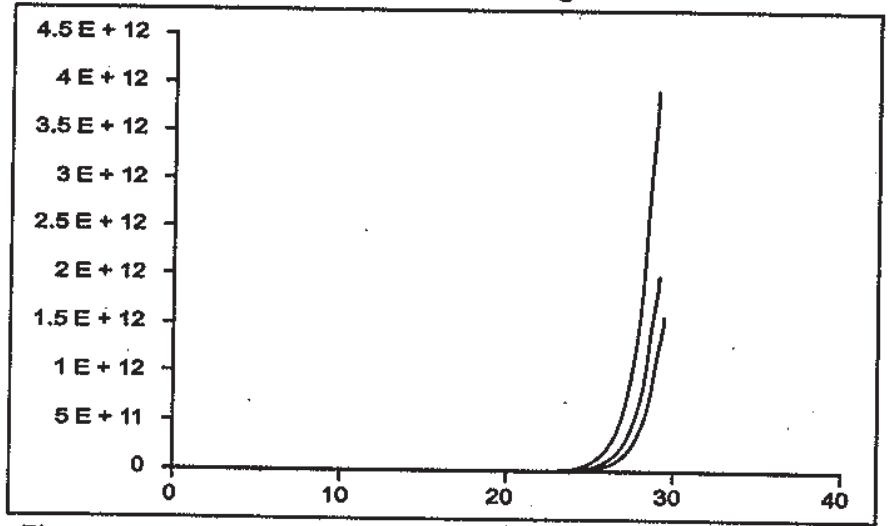

Figure 2. Streamline pattern for $y-C_{1} e^{x}-C_{2} x=$ constant.

Example 3. (Flow with $x\left(y-C_{1} x-C_{2}\right)=$ constant as streamlines). We assume

$$
y=C_{1} x+C_{2}+M(\psi) / x, \quad M^{\prime}(\psi) \neq 0
$$

where $C_{1}, C_{2}$ are arbitrary constants and therefore, we have $g(x)=C_{1} x+C_{2}, \quad f(x)=1 / x$

The streamline pattern for this flow is shown in Figure 3. $\left(C_{1}=0\right)$

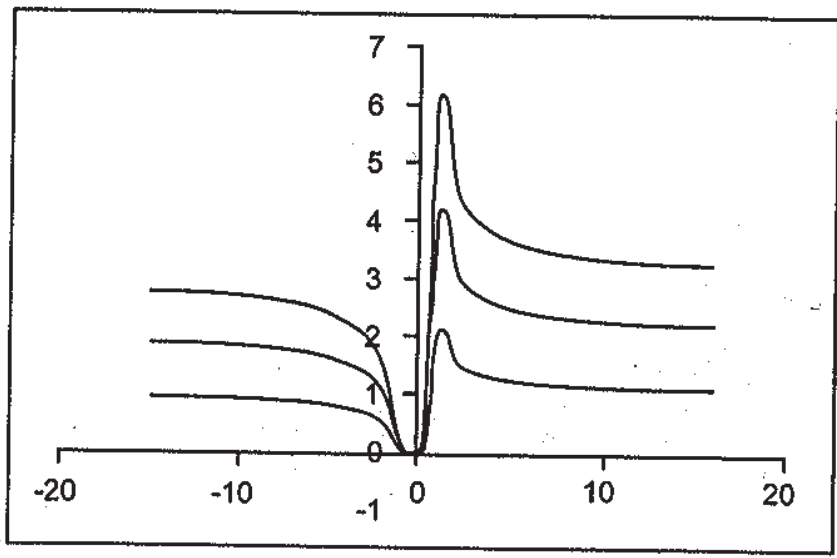

Figure 3. Strecimline pattern for $x\left(y-C_{1} x-C_{2}\right)=$ constant. 
Example 4. (Flow with $y-C_{1} x^{3}-C_{2} x^{2}+C_{3}=$ constant as streamlines). We assume

$$
y=C_{1} x^{3}+C_{2} x^{2}+1+M(\psi) ; \quad M^{\prime}(\psi) \neq 0
$$

where $C_{1}, C_{2}$ and $C_{3}$ are arbitrary constants and therefore, we have

$$
g(x)=C_{1} x^{3}+C_{2} x^{2}+C_{3}, \quad f(x)=1 .
$$

The streamline pattern for this flow is shown in Figure 4.

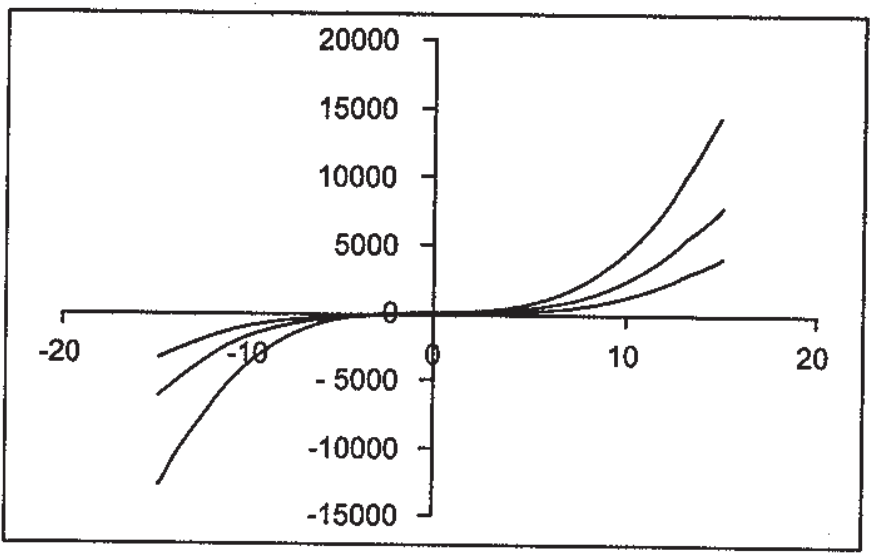

Figure 4. Streamline pattern for $y-C_{1} x^{3}-C_{2} x^{2}+C_{3}=$ constant.

Remark: Similarly we can discuss applications in $(y, \psi)$ net.

\section{References}

1. M.H. Martin, The flow of a viscous fluid. J. Arch. Rat. Mech. Anal., 41, pp 266-286, 1971.

2. O.P. Chandna and F. Labropulu, Exact solutions of steady plane using Von. Mises Coordinates. J. Math. Anal. Appl., 185(1), pp. 36-64, 1994.

3. K.R. Rajagopal, A note on unsteady unidirectional flows of a non-Newtonian fluid, Intemat. J. Non-Linear Mech. 17, 369-373, 1982.

4. F. Labropulu, Generalized Beltrami flows and other closed form solutions of an unsteady viscoelastic fluid, I. J. M.M.S. 30:5, $271-282,2002$.

5. F. Labropulu and O.P. Chandna, Exact solutions of steady plane flows using $(V, \psi)$ coordinotes, Internet. J. Math and Math. Sci. Vol. 23, No.7, 449-475, 2000.

6. C.S. Bagewadi and Siddabasappa, The plane rotating viscous MHD flows, Bull. Cal. Math. Soc, 85, 513-520, 1993.

7. A.M. Siddiqui, P.N. Kaloni and O.P. Chandna, Hodograph transformation methods in non-Newtonion fluids, J. Engg. Math:19, 203-216, 1985. 
8. B.D. Coleman and W. Noll, An approximation theorem for functionals with applications in continuum mechanics, Arch. Rational Mech. Anal. 6, 355-370, 1960.

9. C.S. Bagewadi and S. Bhagya, Behaviour of streamlines in aligned flow, Far East. J. Appl. Math, Vol. 17, No. 2, 121-138, 2004.

10. C. Truesdell and W. Noll, The non-linear field theories of mechanics, in Handbuch der physik, Vol. III/3, Springer, Berlin, 494-513, 1965.

11. Erickson. J.L. "Tensor fields", in Handbuch der physik, Vol. III/3 (ed. S. Flugge), SpringerVerlag, Berlin, 794-858, 1960. 